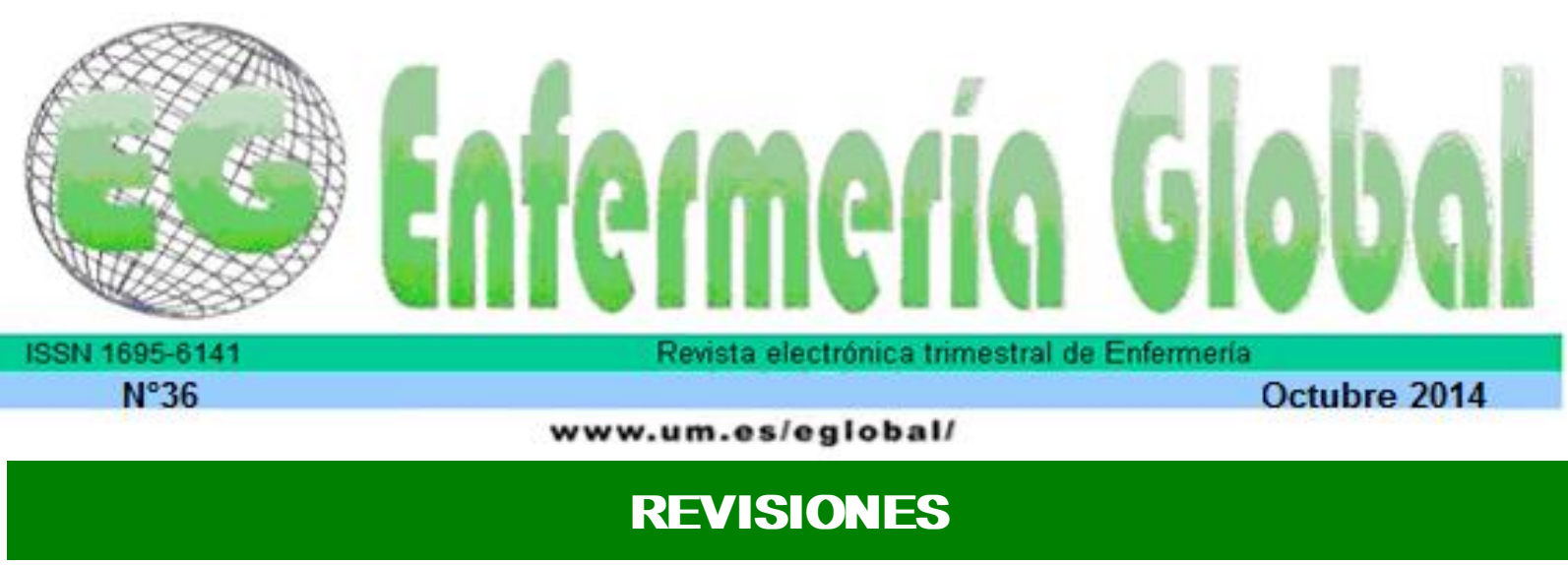

\title{
Morbilidad y mortalidad entre recién nacidos de riesgo: una revisión bibliográfica
}

Morbidade e mortalidade entre recém-nascidos de risco: uma revisão bibliográfica

Morbidity and mortality among the high-risk newborns: a bibliography review

\begin{abstract}
${ }^{*}$ Faria, Cleberson de Souza ${ }^{* *}$ Martins, Christine Baccarat de Godoy ***Lima, Fernanda Cristina Aguiar **Gaíva, Maria Aparecida Munhoz

*Estudiante de Enfermería de la Universidad Federal de Mato Grosso, Cuiabá-MT, Becario de iniciación científica PIBIC - CNPq. ${ }^{* *}$ Postdoctora en Salud Pública por la Facultad de Salud Pública de la USP. Docente del Departamento de Enfermería de la Universidad Federal de Mato Grosso, Cuiabá-MT. E-mail: leocris2001@terra.com.br ***Alumna de Máster en Enfermería de la Universidad Federal de Mato Grosso, Cuiabá-MT, Brasil.
\end{abstract}

\begin{abstract}
Palabras clave: morbilidad; mortalidad neonatal; prematuro; recién-nacido de bajo peso; factores de riesgo
\end{abstract}

Palavras chave: morbidade; mortalidade neonatal; prematuro; recém-nascido de baixo peso; fatores de risco

Keywords: morbidity; neonatal mortality; premature; low weight newborn; risk factors.

\section{RESUMEN}

Ante las necesidades de atención a la salud de los recién nacidos de riesgo, este estudio presenta una revisión bibliográfica sobre la morbimortalidad en este grupo, según las bases de datos LILACS, MEDLINE y SCIELO, en portugués, inglés y español, entre los años 2005 y 2010 . El análisis del contenido permitió organizar el conocimiento en torno al tema en las siguientes categorías: datos epidemiológicos; factores asociados (de riesgo y de protección); evitabilidad del óbito, la importancia de prevención y vigilancia; los servicios de atención al recién nacido de riesgo en Brasil. Se observó que todavía existen desafíos respecto a la reducción de la morbimortalidad entre los recién nacidos de riesgo, dado que el período neonatal constituye uno de los principales indicadores de salud del país, lo que exige la atención de los profesionales y gestores de salud, para la elaboración de políticas específicas.

\section{RESUMO}

Frente às necessidades de atenção à saúde dos recém-nascidos de risco, este estudo apresenta uma revisão bibliográfica acerca da morbimortalidade neste grupo. A busca nas bases de dados LILACS, 
MEDLINE e SCIELO, considerando os anos de 2005 a 2010 e os idiomas português, inglês e espanhol. A análise do conteúdo permitiu organizar o conhecimento a respeito do tema nas seguintes categorias de análise: dados epidemiológicos; fatores associados (de risco e de proteção); a evitabilidade do óbito, a importância da prevenção e vigilância; os serviços de atenção ao recém-nascido de risco no Brasil. Observou-se que, no Brasil, ainda há desafios no que se refere à redução da morbimortalidade entre os recém-nascidos de risco, uma vez que o período neonatal constitui-se um dos principais indicadores de saúde do país, o que demanda atenção dos profissionais e gestores da saúde, com necessidade de elaboração de políticas específicas para o grupo em questão.

\section{ABSTRACT}

Against to the requirements of attention to the health of high-risk newborns, this study presents a review of the published literature on the morbidity and mortality in this group. A search was carried out in the databases LILACS, MEDLINE and SCIELO in the period between 2005 and 2010, publications in English, Spanish and Portuguese were selected. The content analysis enabled us to organize the knowledge into a categories of analysis: epidemiological data; associated factors (risk and protection); evitability of death, the importance of prevention and monitoring; the services of attention to the highrisk newborns in Brazil. We observed that in Brazil, there are challenges about the reduction in morbidity and mortality among the high-risk newborns, since the neonatal period is one of the main child health indicators, which demand attention of professionals and managers of health, the necessity of the establishment of specific policies for the group in question.

\section{INTRODUCCIÓN}

Debido a la vulnerabilidad de la faja etária de menores de un año, la mortalidad infantil es considerada un importante indicador para evaluar la situación de salud de una población y la calidad de las acciones de salud ${ }^{(1-2)}$.

A pesar de la importante reducción de la mortalidad infantil en los últimos años en nuestro país, principalmente con la reducción de la mortalidad postneonatal (de 28 a 364 días de vida), los índices todavía son elevados, considerando que hubo un estancamiento en la mortalidad neonatal $(0 \text { a } 27 \text { días de vida })^{(3-4)}$. En este contexto, destacan los recién nacidos (RN) de riesgo, grupo considerado vulnerable por su alta mortalidad, principalmente en la primera semana de vida. La mortalidad en la primera semana de vida es el componente de la mortalidad infantil de más difícil reducción, pues es resultado tanto de condiciones adversas de la madre como del recién nacido, contribuyendo al aumento del riesgo de muerte y a la necesidad de mayor complejidad de la atención hospitalaria. Muchos autores señalan el bajo peso al nacer y la prematuridad como los principales predictores de mortalidad neonatal ${ }^{(1,4-6)}$.

Estudios resaltan que estas causas, evitables la mayoría de las veces, derivan de una combinación de factores biológicos y ambientales, socio-económicos, culturales y de fallos del sistema de salud o falta de acceso, en los cuales las intervenciones dirigidas a su reducción dependen directamente de mejores condiciones de vida de la población, así como de políticas públicas de salud dirigidas a sus determinantes ${ }^{(1,3,7-9)}$.

Tan importante como la mortalidad, la morbilidad en el grupo de niños en situación de riesgo también se vuelve relevante, ya que puede llevar a la muerte debido a la mayor vulnerabilidad de este grupo, principalmente en los casos de infecciones respiratorias agudas $y$ afecciones perinatales ${ }^{(10)}$.

Según el Ministerio de Salud (MS), deben considerarse neonatos de riesgo los que presenten al menos uno de los criterios siguientes: residencia en área de riesgo, peso al nacer menor de 2.500 gramos, neonato con menos de 37 semanas de gestación, 
asfixia grave (Apgar menor de 7 en el $5^{\circ}$ minuto de vida), hospitalización 0 interocurrencia en la maternidad o en la unidad de asistencia al RN, necesidad de orientación especial en el alta de la maternidad o de la unidad de $\mathrm{RN}$, hijo de madre adolescente (menor de 18 años), madre con baja instrucción (menos de 8 años de estudios) e historial de muerte de niño menor de 5 años en la familia ${ }^{(8)}$.

Frente al impacto de la morbimortalidad entre $\mathrm{RN}$ de riesgo y a la importancia de que los profesionales estén atentos a la cuestión, el presente estudio objetivó presentar una revisión bibliográfica sobre la mortalidad y morbidad en el grupo en cuestión. La importancia de realización de este trabajo se fundamenta en la posibilidad de proporcionar una visión global de la problemática abordada, proporcionando subsidios teóricos sobre el tema.

\section{METODOLOGÍA}

Se trata de una revisión bibliográfica acerca de la morbimortalidad en $\mathrm{RN}$ de riesgo, mediante la busca en bases de datos LILACS, MEDLINE y SCIELO, usando los descriptores DESCs: mortalidad infantil, mortalidad neonatal, morbidad infantil, morbidad, recién nacido de bajo peso, enfermedades del prematuro, prematuro, nacimiento prematuro, recién nacido prematuro, factores de riesgo.

La colecta de datos se realizó de febrero a abril de 2011. Se buscaron publicaciones en el periodo de 2005 a 2010, con la selección de artículos completos, manuales del Ministerio de Salud, tesis y disertaciones disponibles on-line gratuitamente, considerando los idiomas portugués, inglés y español. La elección de la producción de los últimos 05 (cinco) se justifica por el hecho de ser más las actualizadas disponibles sobre el tema.

Las publicaciones se analizaron según la institución de los autores, si publicación nacional o internacional, el año de publicación, el tipo de publicación (artículos completos, tesis, disertaciones, manuales, informes) y el área de conocimiento en que fue publicado.

Los resultados fueron interpretados, primeramente, por medida cuantitativa (frecuencia relativa y absoluta) y, posteriormente, el análisis del contenido permitió organizar el conocimiento respecto al tema a través de categorías de análisis.

\section{RESULTADOS}

Se encontraron 49 trabajos referentes a la morbimortalidad en $\mathrm{RN}$ de riesgo, 47 nacionales $(96,0 \%)$ y 2 internacionales $(4,0 \%)$ (Tabla 1), siendo 38 artículos completos (77,5\%), 03 manuales (6,1\%), 01 documento publicado por el Ministerio de Salud (2,0\%), 01 documento electrónico (conferencia por videotransferencia en formato pdf) (2,0\%), 01 Ordenanza del Ministerio de Salud (2,0\%), 03 disertaciones $(6,1 \%)$ y 02 tesis $(4,1 \%)$. Entre los manuales, 2 de ellos (del Ministerio de Salud) fueron publicados en 2003 y 2004, así como un documento publicado por el Ministerio de Salud en 2004, que fueron incluídos en la investigación debido a su importancia para el estudio.

En relación al vínculo del autor, 39 (79,6\%) estudios fueron desarrollados en instituciones de enseñanza superior, 05 (10,2\%) en órgano oficial (Ministerio de 
Salud), 02 (4,1\%) en servicios de salud y $03(6,1 \%)$ por la Fundación Oswaldo Cruz (tabla 1).

El periodo de publicación de los artículos con mayor frecuencia fue en al año 2005 (22,4\%), seguido de los años 2007 (18,4\%), 2008 (16,3\%), 2010 (14,3\%), 2009 (12,2\%), $2006(10,2 \%), 2004(4,0 \%)$ y $2003(2,0 \%)$ (Tabla 1$).$

Entre los artículos publicados, 32,6\% fueron publicados en periódicos de Salud Pública, 38,8\% en el área de Medicina, 8,2\% en periódicos de Enfermería, 8,2\% en el área de Epidemiología, 2,0\% en el área Social y Económica, y 10,2\% en el área de salud (Ministerio de Salud) (Tabla 1).

Tabla 1 - Distribución de las publicaciones según la nacionalidad, institución de vinculación de los autores, año de publicación y área de conocimiento. Cuiabá, 2011.

\begin{tabular}{lrr}
\hline Nacionalidad & $\mathbf{n}^{\mathbf{0}}$ & $\%$ \\
\hline Nacional & 47 & 96,0 \\
Internacional & 02 & 4,0 \\
Total & 49 & 100,0 \\
\hline Institución de vinculación de los autores & $\mathbf{n}^{\circ}$ & $\%$ \\
\hline Enseñanza superior & 39 & 79,6 \\
Servicio de Salud & 02 & 4,1 \\
Órgano oficial & 05 & 10,2 \\
Fundación Oswaldo Cruz & 03 & 6,1 \\
Total & 49 & 100,0 \\
\hline Año de publicación & $\mathbf{n}^{\circ}$ & $\%$ \\
\hline 2003 & 01 & 2,0 \\
2004 & 02 & 4,0 \\
2005 & 11 & 22,4 \\
2006 & 05 & 10,2 \\
2007 & 09 & 18,4 \\
2008 & 08 & 16,3 \\
2009 & 06 & 12,2 \\
2010 & 07 & 14,3 \\
Total & 49 & 100,0 \\
\hline Área de conocimiento & $\mathbf{n}^{\circ}$ & $\%$ \\
\hline Salud Pública & 16 & 32,6 \\
Medicina & 19 & 38,8 \\
Enfermería & 04 & 8,2 \\
Epidemiología & 04 & 8,2 \\
Social y Económica & 01 & 2,0 \\
Área de salud (Ministerio de Salud) & 05 & 10,2 \\
Total & 05 \\
\hline & 49 & 100,0 \\
\hline
\end{tabular}

\section{DISCUSIÓN}

El análisis del contenido posibilitó organizar el conocimiento producido según las siguientes categorías: datos epidemiológicos; factores asociados (de riesgo y de 
protección); la evitabilidad del óbito, la importancia de la prevención y vigilancia; los servicios de atención al $\mathrm{RN}$ de riesgo en Brasil.

\section{Datos Epidemiológicos}

En las últimas décadas, Brasil viene presentando una importante reducción en la tasa de mortalidad infantil, pasando de 47,1/1000 nacidos vivos en 1990 a 19,3/1000 en 2007, principalmente con la reducción del período postneonatal, mientras que el periodo neonatal a pesar de haber tenido una pequeña caída, mantuvo su tasa elevada ${ }^{(1,3)}$.

En este contexto, la mortalidad neonatal se convirtió en el principal componente de la mortalidad infantil al inicio de los años 90 , representando del $60 \%$ al $70 \%$ de los óbitos en menores de 1 año en Brasil y cerca del $75 \%$ en el mundo ${ }^{(1-3,5,11)}$, por lo que la necesidad de inversiones tecnológicas más avanzadas, incluyendo el acceso al servicio de mayor complejidad, en el caso de las unidades neonatales de cuidados intensivos, contribuyen a la persistencia de ese elevado índice ${ }^{(12-13)}$.

Datos de DATASUS muestran que en 2007, en Brasil, la mortalidad neonatal fue de 13,6 por 1.000 nacidos vivos. Si comparamos este dato con la tasa de otros países como Chile (5/1000), Canadá (3/1000), Cuba (4/1000) y Francia (2/1000), se percibe que la tasa de mortalidad neonatal en Brasil sigue siendo elevada ${ }^{(3,14)}$.

En cuanto a las causas de esos óbitos, las afecciones perinatales corresponden al $80 \%$ de los óbitos neonatales, destacando la prematuridad y sus complicaciones (como la dificultad respiratoria del recién nacido o enfermedad de la membrana hialina $)^{(3)}$.

Para algunos autores, además de las afeccioneses perinatales y la prematuridad, la asfixia intrauterina y el intraparto y el bajo peso al nacer constituyen las principales causas de óbitos neonatales ${ }^{(12)}$.

En estudio realizado por Duarte y Mendonça (2005a), en 4 maternidades de Rio de Janeiro, en 2005, con $507 \mathrm{RN}$ de riesgo, se identificó como principales causas de muerte en los primeros seis días, por orden de frecuencia, la enfermedad de la membrana hialina, la sepsis, la hemorragia pulmonar, la asfixia perinatal, pneumotórax y la neumonía congénita; mientras que en el periodo entre 7 y 27 días de vida la causa de óbito más frecuente fue la sepsis, seguida de la neumonía congénita.

Los datos epidemiológicos muestran que hay mucho que avanzar para la reducción de la morbimortalidad de los $\mathrm{RN}$ de riesgo. El actual contexto refleja la relevancia de una mayor atención para la promoción de la salud del grupo en cuestión, necesitando de inversiones y/o estrategias que disminuyan el impacto negativo en la salud pública, alcanzando de esta forma índices cada vez menores de morbimortalidad, caminando en busca de niveles semejantes a los de los países desarrollados.

\section{Factores asociados de riesgo y de protección}

La literatura apunta algunos factores como determinantes para la morbimortalidad neonatal, como los relacionados con el nivel socio-económico, con el recién nacido, 
con la historia reproductiva/obstétrica materna y con la disponibilidad y calidad de la atención perinatal $^{(2,9)}$.

Un estudio ${ }^{(15)}$ identificó entre las características socioeconómicas, tales como la escolaridad del cabeza de familia, el número de habitaciones y la localización del domicilio como factores de riesgo asociados negativamente a la mortalidad neonatal precoz, asociados a la baja renta familiar ${ }^{(9)}$. Posiblemente, estos factores se explican en función de las malas condiciones de habitabilidad que hacen al RN más vulnerable a los problemas de salud. Estudios apuntan el lugar de residencia como factor de riesgo relacionado con la morbimortalidad neonatal, pues la localización de la vivienda puede estar asociada a la cuestión del acceso a los servicios de salud, ya que si consideramos la estructura de atención, localización y calidad de los servicios, el acceso a los bienes y servicios de salud es restringido, como relata investigación ${ }^{(16)}$ en que el $52 \%$ de la población estudiada residía en zona rural y/o periferia.

Estudio revela características psicosociales maternas asociadas a los óbitos neonatales, tales como madre adolescente y presencia de violencia doméstica, y también observaron que, entre los factores biológicos y de historia reproductiva materna, destacan las interocurrencias durante la gravidez, adecuación de prenatal, tabaquismo en la gestación y nacimiento previo de bajo peso en los óbitos neonatales. El mismo trabajo también presenta prenatal ausente o inadecuado como factor de riesgo asociado a la mortalidad neonatal precoz ${ }^{(15)}$.

Autores señalan que el peso al nacer es, aisladamente, el principal factor de riesgo asociado a la morbimortalidad neonatal. La prematuridad, edad materna, gravidez en la adolescencia, desnutrición intrauterina y deficiencia en la asistencia prenatal son considerados factores de riesgo para el bajo peso al nacer con el consecuente aumento en la morbimortalidad neonatal, en una relación inversamente proporcional, o sea, el riesgo de muerte entre RNs de riesgo aumenta en la medida en que el peso en el nacimiento disminuye ${ }^{(17-18)}$.

Respecto a la prematuridad, los principales factores de riesgo asociados al bajo peso y también a la morbimortalidad neonatal son: la edad materna joven o avanzada, educación y nivel socioeconómico de la madre, tabaquismo, múltiples gestaciones, metrorragia en los $2^{\circ}$ y $3^{\circ}$ trimestres, infecciones maternas, oligodramnios/polidramnios y patologías de la madre como diabetes e hipertensión ${ }^{(19)}$.

Destacan también los RNs de muy bajo peso, pues además de representar la mitad del número de muertes en el periodo neonatal, llegan a presentar un riesgo de óbito 200 veces mayor en relación al $\mathrm{RN}$ con peso superior a $2.500 \operatorname{gramos}^{(2,20)}$.

Entre los factores de protección, el uso de corticosteroide prenatal (medicamento que contiene corticoide que acelera la maduración pulmonar, acelerando el desarrollo alveolar y estimulando la secreción de surfactante) se mostró como uno de los más importantes contra la morbidad y mortalidad neonatal, pues se trata de un procedimiento profiláctico contra el problema respiratorio, principalmente en lo que se refiere a la membrana hialina, siendo menos oneroso y de más fácil utilización, presentando mejores resultados que el uso de la oxigenoterapia con respiradores artificiales y surfactante pulmonar ${ }^{(9,21)}$.

Sin embargo, autores llaman la atención respecto al uso de los corticosteroides, pues estos deben ser evitados tras el nacimiento, optándose por el surfactante, pues el uso 
continuo de los corticoides puede causar complicaciones tales como perforación gastrointestinal, hipertensión, hiperglicemia, sepsis, déficit en el crecimiento y alteraciones en el desarrollo neurológico ${ }^{(19)}$.

Además del uso de corticosteroide prenatal, tener una asistencia prenatal adecuada y ser primigesta, se mostraron como factores asociados a la disminución de la mortalidad neonatal, pues influyen positivamente en la madurez, aumento de peso y desarrollo intrauterino saludable del $\mathrm{RN}$ de riesgo ${ }^{(9,15)}$.

Otro aspecto importante para la protección de los $\mathrm{RNs}$ de riesgo, en lo que se refiere a la morbimortalidad, es la escolaridad materna. La mayor escolaridad en la educación femenina asociada a las medidas de prevención y promoción de la salud permite mayor acceso a las informaciones de salud ${ }^{(16,18)}$.

De esta manera, la educación y la formación informal, a través de la cual se puede realizar promoción de conocimientos y prácticas de salud, aliadas a la escuela y a otras instituciones, promueven la búsqueda de transformaciones sociales ${ }^{(16)}$. En este sentido el conocimiento materno puede influir tanto en la elección de métodos anticonceptivos como en la propia planificación familiar, y, además de esto, la mayor escolaridad contribuye a la formación de una consciencia preventiva en relación a la salud y enfermedad, desde la higiene personal hasta cuidados con la propia gestación, siendo considerada como un relevante factor tanto para la salud materna como para la infantil, principalmente de los $\mathrm{RN}$ de riesgo ${ }^{(18)}$.

\section{La evitabilidad del óbito, la importancia de la prevención y vigilancia}

Algunas causas de óbito en el periodo neonatal se consideran evitables por medio de diagnóstico e intervención precoces y otras, incluso, son consideradas reducibles a partir del adecuado acompañamiento de la gestación y del parto ${ }^{(7)}$.

El Ministerio de Salud (MS) propuso una lista de causas de muerte evitables por intervenciones del SUS, reducibles por acciones de inmunoprevención, atención a la mujer en la gestación y en el parto, atención al recién nacido, acciones de diagnóstico, tratamiento, promoción y atención a la salud ${ }^{(3)}$.

Si tomamos en consideración la lista propuesta por el MS, observamos que los principales factores de riesgo asociados a la morbimortalidad de los $\mathrm{RNs}$ de riesgo, de hecho podrían ser evitados por acciones de prevención y promoción de la salud, o sea, estos óbitos neonatales podrían evitarse o reducirse si se garantizase el acceso adecuado a los servicios de salud y a las medidas sanitarias de buena calidad ${ }^{(7)}$.

De esta forma, se puede afirmar que la evitabilidad del óbito neonatal está relacionada más con las condiciones de asistencia al prenatal y al parto que a la existencia de sofisticada tecnología de terapia intensiva ${ }^{(7)}$.

Hay que se considerar, por ello, que la atenión terciaria, es decir, las Unidades de Cuidados Intensivos Neonatales (UCIN) son coadyuvantes importantes en los cuidados prestados a los neonatos de riesgo, pues dispone de tecnologías avanzadas, proporcionando diferentes modalidades de ventilación mecánica, monitorización y cirugía neonatal que garanticen la supervivenci del grupo en cuestión $^{(19)}$. 
En este contexto la focalización de las políticas de salud para la reducción de óbitos en el período neonatal y neonatal precoz tienen resultado en la implantación y aumento de la oferta de $\mathrm{UCIN}^{(11)}$. En los últimos años, hubo un aumento en el número de camas intermedias y de terapia intensiva neonatal en el sector público, principalmente en las regiones metropolitanas del Sur y Sudeste ${ }^{(11)}$. Este cambio ya fue observado en el municipio de Rio de Janeiro, que incorporó maternidades estaduales y federales, aumentando el número de camas hospitalarias para gestantes y recién nacidos de alto riesgo. Para esto, fue necesario un aumento de los recursos materiales, así como el uso de nuevas tecnologías para tratamiento, tanto de las gestantes como de los recién nacidos de riesgo, además de la formación de recursos humanos $^{(2)}$.

La implantación de unidades de cuidados intensivos proporciona la supervivencia de recién nacidos cada vez más prematuros y de bajo peso, por ello, incluso con cuidados específicos, aún se verifica gran proporción de óbitos en este grupo ${ }^{(11)}$.

Ante el panorama nacional de buscar estrategias que posibiliten la prevención y reducción de los óbitos infantiles, principalmente en el periodo neonatal, hay que destacar la creación de los Comités de Prevención del Óbito Infantil y Fetal ${ }^{(3)}$.

La implantación de estos comités en Brasil tuvo las primeras iniciativas a mediados de la década de los 90, sin embargo, esta estrategia solo se hizo realidad tras la creación del Manual de Vigilancia del Óbito Infantil y Fetal y del Comité de Prevención del Óbito Infantil y Fetal, elaborado en 2004, que sirve de referencia para la estructuración de los comités, proporcionando subvenciones y orientaciones para su funcionamiento y para la vigilancia de los óbitos, así como disponibilizando modelos actualizados de formularios de investigación y análisis ${ }^{(3)}$.

Con la elaboración de este manual fue posible sistematizar las experiencias de vigilancia del óbito infantil y fetal, movilizando así la participación y compromiso de los gestores y profesionales de salud, a fin de organizar un sistema de acompañamiento de los óbitos con mayor potencial de prevención ${ }^{(3)}$.

De acuerdo con el MS, el $50 \%$ de los municipios con población de 80.000 habitantes o más ya tienen comités, con una proporción mayor en las regiones Sur y Sudeste, confirmando la vigilancia de la mortalidad infantil y fetal como una de las prioridades del $\mathrm{MS}^{(3)}$.

Aún hay mucho que avanzar y mejorar en lo que se refiere a la prevención, evitabilidad y reducción de los óbitos neonatales. Sin embargo, esto solamente será posible con el compromiso de las autoridades y, principalmente, de los gestores y profesionales del área de la salud, pues para cambiar la actual realidad es preciso trabajar para que los resultados conquistados puedan tener el mayor impacto positivo posible.

\section{Servicios de Atención al Recién Nacido de Riesgo en Brasil}

En Brasil, son escasos los estudios sobre el efecto de los servicios de salud en la mortalidad neonatal. En diversos estudios se ha demostrado la importancia del acceso y calidad de la asistencia prenatal sobre la mortalidad perinatal, por eso hay pocas investigaciones sobre asistencia neonatal ${ }^{(20)}$. 
Durante muchas décadas, todos los esfuerzos estuvieron orientados, casi exclusivamente, en la mejora de la asistencia prenatal, con menor énfasis en medidas como la continuidad de la asistencia hasta el momento del parto y la asistencia hospitalaria cualificada, por lo que para la reducción de la morbidad y consecuentemente de la mortalidad neonatal, se torna prioritaria la inversión en la reestructuración de la asistencia a la gestante y al recién nacido, con articulación entre atención básica (prenatal) y asistencia al parto ${ }^{(20)}$.

Al haber pocos estudios sobre la asistencia neonatal, es posible afirmar que hasta la década de los 90 no había políticas específicas para el cuidado del $\mathrm{RN}$ de riesgo. Destaca el servicio de atención básica, al cual se incorporaron acciones específicas en estrategias de refuerzo, para presentar un nuevo patrón de modelo asistencial, como el Programa de Agentes Comunitarios de Salud (PACS) y, posteriormente, el Programa Salud de la Familia (PSF), apuntando la necesidad de un trabajo amplio e integrado, incluyendo acciones destinadas a mejorar la atención prestada y a reducir la morbimortalidad en la infancia ${ }^{(10)}$.

Sin embargo, incluso el PSF, hoy Estrategia de Salud de la Familia (ESF), no presentaba ninguna programación específica para el acompañamiento de los RNs de riesgo, lo que vino a convertirse en realidad en 2004, cuando tuvo lugar el lanzamiento de la Agenda de Compromisos para la Salud Integral del Niño y Reducción de la Mortalidad Infantil ${ }^{(22)}$.

Esta agenda buscó organizar la asistencia de salud en líneas de cuidado, una estrategia para superar la desarticulación entre los diversos niveles de atención en salud (como el distanciamiento entre la atención básica y la atención hospitalaria) y garantizar la continuidad del cuidado integral del usuario, con acciones de promoción, tratamiento y rehabilitación, de manera ágil y oportuna en cada nivel de atención (primaria, secundaria y terciaria), con referencia y contra-referencia responsable, hasta la recuperación completa del individuo ${ }^{(8)}$.

Dentro de las líneas de cuidado, destacan la atención integral de la salud del niño (priorizándose el acompañamiento del $\mathrm{RN}$ de riesgo), la reducción de la mortalidad infantil, la atención humanizada y cualificada a la gestante y al RN e incentivo de la lactancia materna ${ }^{(8)}$.

Hay que destacar la Primera Semana de Salud Integral en la Unidad Básica de Salud (UBS), en la que se realizan visitas domiciliarias en los primeros 7 (siete) días, y a través de las informaciones recogidas en la cartilla del niño (que debe ser entregada por la maternidad al nacimiento del niño), prioriza el cuidado y acompañamiento del $\mathrm{RN}$ de riesgo, considerando que la primera semana es el periodo en que más se concentran los óbitos neonatales ${ }^{(8,20)}$.

En este sentido, para promover una mejor calidad en la asistencia neonatal y reducción de la morbimortalidad de este grupo, en 2006, el MS, a través de la Ordenanza MS/GM n. 399 23, aprobó el Pacto por la Salud, estableciendo entre sus prioridades el Pacto por la vida y la reducción de la mortalidad materna, neonatal e infantil ${ }^{(23)}$.

Se resalta que desde 2004 ya había sido discutida y aprobada por la Comisión Intergestores TRIPARTITA una versión del Pacto Nacional Por la Reducción de la 
Mortalidad Materna y Neonatal que sería confirmado por la Ordenanza MS/GM n. $399^{(23)}$.

En lo que se refiere a los neonatos de riesgo, el pacto presentaba las siguientes propuestas/objetivos: la expansión de la atención básica; lanzamiento de la Primera Semana de Salud Integral; organización del acceso (integrar los niveles de atención, garantizando la continuidad del cuidado, camas de $\mathrm{UCl}$, y transferencia en situación de riesgo); adecuación de la oferta de servicios (ampliar el registro de camas de UCI neonatal) y priorización en el costo de los servicios, definiendo como prioridad en los procesos presupuestarios de unidades hospitalarias aquellas que tienen un proceso de referencia regional para la atención a la gravidez de riesgo y que dispongan de $\mathrm{UCIN}^{(22)}$.

Del mismo modo que en la atención básica, es esencial realizar inversiones en la atención terciaria, como nos muestran autores ${ }^{(2)}$, que observaron en su estudio el impacto de la mejora en la asistencia al parto, aumento del número de camas, elevación de recursos materiales con tecnologías modernas, estandarización de la conducta en la atención, obligatoriedad de la presencia del pediatra en la sala de parto y el aumento y formación de los profesionales involucrados en la asistencia en la reducción de la mortalidad de neonatos de riesgo.

En este contexto, es fundamental que la atención básica y la atención terciaria caminan articuladas entre sí, pues todos los niveles de atención a la salud deben trabajar de modo integrado, a fin de proporcionar un cuidado adecuado, de calidad e integral a los neonatos de riesgo, para que desta forma, alcancemos una realidad mejor en lo que se refiere a la salud del periodo neonatal en nuestro país.

\section{CONCLUSIÓN}

Frente a los resultados encontrados, Brasil todavía enfrenta desafíos en lo que se refiere a la asistencia a la salud de los $\mathrm{RNs}$ de riesgo y reducción de la morbimortalidad en el grupo. Por estar incluído en uno de los principales indicadores de salud de Brasil, el periodo neonatal merece más atención por parte de los profesionales y gestores del área de salud, en especial en lo tocante al nacimiento de riesgo.

Al detectar que la mayoría de los problemas y óbitos pueden ser evitados, prevenidos y reducidos, reconocemos la relevancia de la mejora en la asistencia prenatal y de la continuidad de atención de calidad durante el parto y tras el nacimiento.

Así, la atención básica y las UCINs desempeñan un importante papel para la promoción del cuidado a los neonatos de riesgo. De la misma forma que es preciso una atención mayor a los $\mathrm{RNs}$ de riesgo en la atención básica de salud, desarrollando un programa propio dentro de este servicio, como en la salud de la gestante y anciano por ejemplo, es necesario inversión para el aumento del número de camas de las UCINs, así como la formación, perfeccionamiento y educación continua de los profesionales de esas UCIN y de la Estrategia Salud de la Familia.

A pesar de los avances observados en los últimos años en lo que se refiere al período neonatal a través de políticas como la Agenda de Compromisos para la Salud Integral del Niño y Reducción de la Mortalidad Infantil y el Pacto Nacional Por la Reducción de 
la Mortalidad Materna y Neonatal, Brasil tiene pocos servicios y políticas específicas para la asistencia a los neonatos de riesgo.

Se destaca también la necesidad de incluir el tema en los cursos de salud, tanto a nivel técnico como de graduación y posgraducación, a fin de contemplar la temática en la formación de los profesionales.

Otra consideración importante se refiere a la calidad de la información que alimenta las estrategias de vigilancia del grupo en cuestión. Para ello, es importante llamar la atención en cuanto a la valorización que debe ser dada por los profesionales de salud a la cumplimentación de forma correcta de los formularios de declaraciones de óbitos, para alimentar de forma correcta la vigilancia epidemiológica, a fin de contribuir a que las medidas e intervenciones a ser elaboradas sean cada vez más efectivas.

Aumenta también la necesidad de políticas y estrategias de acompañamiento sistematizado de los $\mathrm{RN}$ de riesgo, a fin de evitar la morbidad y la mortalidad elevadas.

Otro factor importante a ser considerado para las transformaciones necesarias referentes a la salud del grupo de los RNs de riesgo es el contexto socioeconómico, o seja, es fundamental ofrecer mejores condiciones de vida para la población, principalmente a las madres, ya que los indicadores de salud está íntimamente relacionados con las condiciones de vida. Además, destacamos la importancia de la educación, pues en la mayoría de los estudios encontrados se identificó que la mayor escolaridad materna se caracteriza como uno de los principales factores de protección de los RNs de riesgo, demostrando que es esencial la inversión en esta área.

Esperamos que el presente estudio, al presentar el contexto actual de la morbimortalidad y de la asistencia prestada a los RNs de riesgo, pueda contribuir a la comprensión del fenómeno en este grupo, sirviendo como punto de partida para estrategias y acciones específicas. Se sugiere, también, la realización de otros estudios, pues hay pocas publicaciones que traten especifícamente de los RNs de riesgo, para que así puedan complementarse las lagunas de conocimiento en el área.

\section{REFERENCIAS}

1. Araújo BF, Tanaka ACA, Madi JM, Zatti H. Estudo da mortalidade de recémnascidos internados na UTI neonatal do Hospital Geral de Caxias do Sul, Rio Grande do Sul. Rev. Bras. Saúde Matern. Infant 2005; 5 (4): 463-9.

2. Duarte JLMB, Mendonça, GAS. Avaliação dos óbitos neonatais em recém-nascidos de muito baixo peso em quatro maternidades no município do Rio de Janeiro, Brasil. Cad. Saúde Pública 2005a; 21(2):387-95.

3. Brasil. Ministério da Saúde. Manual de vigilância do óbito infantil e fetal e do comitê de prevenção do óbito infantil e fetal. $1^{\underline{a}}$ edição. Brasília: Editora do Ministério da Saúde, 2009. 79 p.

4. Silva ZP, Almeida MF, Ortiz LP, Alencar GP, Alencar AP, Schoeps D, et al. Morte neonatal precoce segundo complexidade hospitalar e rede SUS e não-SUS na região metropolitana de São Paulo, Brasil. Cad. Saúde Pública 2010; 26(1):123-34.

5. Giglio MRP, Lamounier JA, Morais Neto OL, César CC. Baixo peso ao nascer em coorte de recém-nascidos em Goiânia-Brasil no ano de 2000. Rev Bras Ginecol Obstet 2005; 27(3): 130-6. 
6. Araújo DMR, Pereira NL, Kac G. Ansiedade na gestação, prematuridade e baixo peso ao nascer: uma revisão sistemática da literatura. Cad. Saúde Pública 2007; 23(4):747-56.

7. Caldeira AP, França E, Perpétuo IHO, Goulart EMA. Evolução da mortalidade infantil por causas evitáveis, Belo Horizonte, 1984-1998. Rev Saúde Pública 2005; 39(1):67-74.

8. Brasil. Ministério da Saúde. Agenda de compromissos para a saúde integral da criança e redução da mortalidade infantil. $1^{a}$ edição. Brasília: Editora do Ministério da Saúde, 2004. 80 p.

9. Duarte JLMB, Mendonça GAS. Fatores associados à morte neonatal em recémnascidos de muito baixo peso em quatro maternidades no Município do Rio de Janeiro, Brasil. Cad. Saúde Pública 2005b; 21(1):181-91.

10. Brasil. Ministério da Saúde. AIDPI Atenção Integrada às Doenças Prevalentes na Infância: curso de capacitação: introdução: módulo 1. 2. edição rev. 1. a reimpressão. Brasília: Editora Ministério da Saúde, 2003. 32p.

11. Assis HM, Machado CJ, Rodrigues RN. Perfis de mortalidade neonatal precoce: um estudo para uma Maternidade Pública de Belo Horizonte (MG), 2001-2006. Rev Bras Epidemiol 2008; 11(4): 675-86.

12. Pedrosa LDCO, Sarinho SW, Ordonha MAR. Óbitos neonatais: por que e como informar? Rev. Bras. Saúde Matern. Infant 2005; 5(4): 411-8.

13. Gomes MASM, Lopes JMA, Moreira MEL, Gianini NOM. Assistência e mortalidade neonatal no setor público do município do Rio de Janeiro, Brasil: uma análise do período 1994/2000. Cad. Saúde Pública 2005; 21(4):1269-77.

14. Datasus. Ministério da Saúde. Informações em Saúde. Indicadores de Saúde. Indicadores de mortalidade. Taxa de mortalidade neonatal precoce e tardia. 2007. [citado em: 11 mai. 2011]. Disponível em < http://tabnet.datasus.gov.br/cgi/idb2009/c0101b.htm> <http://tabnet.datasus.gov.br/cgi/idb2009/c0102b.htm>.

15. Schoeps D, Almeida MF, Alencar GP, França Jr I, Novaes HMD, Siqueira AAF, et al. Fatores de risco para mortalidade neonatal precoce. Rev Saúde Pública 2007; 41(6):1013-22.

16. Ramos HAC, Cuman RKN. Fatores de risco para prematuridade: pesquisa documental. Esc Anna Nery Rev Enferm 2009; 13 (2): 297-304.

17. Malveira SS, Moraes AN, Chermont AG, Costa DLF, Silva TF. Recém-nascidos de muito baixo peso em um hospital de referência. Revista Paraense de Medicina 2006; 20(1): 58-62.

18. Uchimura TT, Pelissari DM, Soares DFPP, Uchimura NS, Santana RG, Moraes CMS. Cienc Cuid Saude 2007; 6(1):51-8.

19. Hübner ME, Nazer J, León GJ. Estrategias para mejorar la sobrevida del prematuro extremo. Rev Chil Pediatr 2009; 80(6): 551-9.

20. Araujo NSA. O papel da assistência hospitalar ao recém-nascido na mortalidade neonatal precoce na Região Sul do município de São Paulo: estudo caso-controle [Tese]. São Paulo: Universidade de São Paulo, Faculdade de Medicina; 2006.

21. Ramos JGL, Martinez FE. Videoconferência. Terapia antenatal com corticóide para maturação fetal: uma importante estratégia de redução da mortalidade neonatal. Brasília: 2010.

22. Brasil. Ministério da Saúde. Secretaria de Atenção à Saúde. Departamento de Ações Programáticas Estratégicas. Pacto Nacional Pela Redução da Mortalidade Materna e Neonatal. Brasília, 2004b. 14 p.

23. Brasil. Ministério da Saúde. Divulga o Pacto pela Saúde 2006 - Consolidação do SUS e aprova as Diretrizes Operacionais do Referido Pacto. Portaria n. 399 de 22 de fevereiro de 2006. 
ISSN 1695-6141

๑ COPYRIGHT Servicio de Publicaciones - Universidad de Murcia 